\title{
Central Tendon of the Diaphragm
}

National Cancer Institute

\section{Source}

National Cancer Institute. Central Tendon of the Diaphragm. NCI Thesaurus. Code C34119.

A crescent-shaped, flat aponeurosis composed of dense collagen fibers that forms the intermediate region of the diaphragm, and blends superiorly with the fibrous pericardium. 